\title{
Alocação de locomotivas no transporte ferroviário de cargas com foco em redução de custos e emissões de $\mathrm{CO}_{2}$
}

No contexto do transporte ferroviário de cargas, a alocação de locomotivas se destaca por ser uma operação estratégica para as empresas do setor em função do elevado investimento necessário para aquisição dos diversos custos relacionados e dos impactos ambientais decorrentes de sua utilização. Logo, em um cenário de crescimento da participação do modo ferroviário na economia brasileira, um maior aprimoramento dos meios de gestão e programação, principalmente através de ferramentas que busquem otimização dos processos, torna-se cada vez mais importante quando se buscam vantagens competitivas frente aos demais concorrentes do setor de transportes. Sendo assim, este artigo apresenta um procedimento para direcionar a decisão de alocação de diferentes modelos de locomotivas em diversos tipos de trens no transporte ferroviário de cargas que visa à redução dos custos do transporte associado a uma diminuição das emissões de $\mathrm{CO} 2$. Foi realizada a aplicação do modelo proposto em uma empresa do setor de transporte ferroviário de cargas brasileiro, gerando uma redução de cerca de $10 \%$ nos custos operacionais e $7 \%$ nas emissões de $\mathrm{CO} 2$, quando comparado com o cenário atual praticado pela empresa. Através da análise dos resultados da aplicação realizada, foi possível constatar a consistência e aplicabilidade do procedimento proposto neste trabalho.

\section{Allocation of rail freight locomotives with focus on cost reduction and $\mathrm{CO}_{2}$ emissions}

\begin{abstract}
In the context of rail freight, locomotive allocation stands out as a strategic operation for companies in the sector due to the high investment required to acquire the various related costs and the environmental impacts arising from their use. Therefore, in a scenario of growth of the participation of the rail mode in the Brazilian economy, a better improvement of the management and programming means, mainly through tools that seek process optimization, becomes increasingly important when looking for competitive advantages over other competitors in the transport sector. Thus, this paper presents a procedure for directing the decision to allocate different locomotive models on different types of trains in rail freight transportation that aims to reduce transportation costs associated with a reduction in $\mathrm{CO} 2$ emissions. The proposed model was applied to a company in the Brazilian rail freight sector, generating a reduction of about $10 \%$ in operating costs and $7 \%$ in $\mathrm{CO} 2$ emissions, when compared to the current scenario practiced by the company. Through the analysis of the results of the application, it was possible to verify the consistency and applicability of the procedure proposed in this work.
\end{abstract}

Keywords: Allocation; Locomotives; Rail transport.

Topic: Sustentabilidade nos Transportes

Reviewed anonymously in the process of blind peer.
Received: $12 / 06 / 2019$

Approved: 14/07/2019
Caio Almeida Arêas Reis (D)

Instituto Militar de Engenharia, Brasil http://lattes.cnpq.br/9412926613962693 http://orcid.org/0000-0003-3876-0796

caioaareis@gmail.com

Renata Albergaria de Mello Bandeira (iD Instituto Militar de Engenharia, Brasil http://lattes.cnpq.br/4038930409521817 http://orcid.org/0000-0002-2776-2473

re.albergaria@gmail.com

Luiz Antônio Silveira Lopes (iD

Instituto Militar de Engenharia, Brasil http://lattes.cnpq.br/4190993496892481 http://orcid.org/0000-0002-2163-9204 laslopes@ime.eb.br
Orivalde Soares da Silva Júnior (DD

Instituto Militar de Engenharia, Brasil http://lattes.cnpq.br/6743760911756716 http://orcid.org/0000-0003-0743-8024 orivalde@yahoo.com.br
Referencing this:

REIS, C. A. A.; BANDEIRA, R. A. M.; LOPES, R. A. M.; LOPES, L. A. S.; SILVA JÚNIOR, O. S. S.. Alocação de locomotivas no transporte ferroviário de cargas com foco em redução de custos e emissões de $\mathrm{CO}_{2}$. Revista Ibero-Americana de Ciências Ambientais, v.10, n.4, p.149-166, 2019. DOI: http://doi.org/10.6008/CBPC2179$\underline{6858.2019 .004 .0012}$ 


\section{INTRODUÇÃO}

O modo ferroviário apresenta como vantagem a sua capacidade de transporte, principalmente quando se faz necessário percorrer longas distâncias, além de se destacar por seus custos ambientais e sociais (CNT, 2013). O transporte ferroviário apresenta menor impacto ambiental em comparação ao modo rodoviário, em decorrência da menor emissão de poluentes e gases de efeito estufa (GEE). Ainda, o nível de segurança do modo ferroviário também é superior em comparação ao rodoviário, em função do menor risco de acidentes envolvendo terceiros ou as próprias locomotivas (CNT, 2013).

A participação do modo ferroviário na matriz de transporte de carga no Brasil cresceu 43,3\% entre 2006 e 2016, atingindo o patamar de 503,8 milhões de toneladas úteis transportadas (CNT, 2017). Entretanto, uma gestão mais eficiente dos ativos operacionais é fundamental para um maior crescimento do setor. Ganhos na otimização de processos que se traduzam em menores custos acabam sendo refletidos na capacidade das empresas de oferecer serviços de qualidade ao mercado, atraindo novas demandas para transporte e gerando assim um diferencial competitivo em um setor tão concorrido.

Neste contexto, as locomotivas devem ter um tratamento diferenciado em função dos seus altos custos de aquisição e manutenção e de sua importância no contexto ferroviário. A sua alocação de forma eficiente influencia diretamente os custos operacionais, contribuindo para aumentar a eficiência da ferrovia (CASSEMIRO, 2017), em especial no cenário brasileiro.

Devido ao modelo de concessões ferroviárias no país, foram herdadas locomotivas da antiga Rede Ferroviária Federal (RFFSA) que, de maneira geral, são frotas bastante diversificadas tanto pelos veículos utilizados quanto pelo tempo de uso dos ativos. Além disto, a instabilidade econômica do país impacta não somente nos investimentos em novas locomotivas, mas também na disponibilidade de veículos, que podem ser empregados de forma mais eficiente para determinados tipos de cargas e composições ferroviárias (CASSEMIRO et al., 2015). Assim, estes diferentes tipos de veículos ferroviários influenciam na alocação de locomotivas.

Deste modo, é fundamental que a alocação das locomotivas nos trens seja realizada da melhor forma possível através da realização de um planejamento eficiente das operações nos níveis estratégico, tático e operacional, configurando um dos principais impulsionadores para tornar as ferrovias cada vez mais competitivas. Ainda convém ressaltar que a gestão eficiente das locomotivas não gera apenas benefícios de redução de custos, mas também ganhos ambientais, como a redução do consumo de combustível e a consequente diminuição da emissão de gases efeito estufa, principalmente o dióxido de carbono $\left(\mathrm{CO}_{2}\right)$ resultante da queima de óleo diesel pelas locomotivas. Sendo assim, o presente artigo apresenta uma proposta de modelo de alocação de locomotivas em trens de carga, com foco na redução dos custos do transporte e da redução da emissão de $\mathrm{CO}_{2}$.

Após esta seção de introdução, este artigo está assim estruturado: na Seção 2 é apresentado o conceito de alocação de locomotivas, bem como uma revisão da literatura sobre os modelos matemáticos propostos para alocação de locomotivas; a Seção 3 apresenta o conceito de eficiência energética e os 
impactos ambientais relacionados; a Seção 4 propõe-se um modelo matemático de alocação de locomotivas, enquanto que na Seção 5 são analisados os resultados da aplicação do modelo proposto com base em dados levantados de uma ferrovia brasileira de transporte de cargas. A Seção 6 apresenta as principais conclusões do trabalho, bem como sugestões de pesquisas futuras.

\section{REVISÃO TEÓRICA}

\section{Alocação de locomotivas}

O processo de programação de locomotivas, segundo Ahuja et al. (2005), consiste em alocar um conjunto de máquinas para atender aos trens programados em um dado horizonte de tempo, garantindo o fornecimento de tração suficiente para os trens e determinando as rotas de todas as locomotivas desde a origem até o destino. Desta forma, se faz necessário considerar o número de locomotivas existentes em cada tipo de frota, as necessidades de abastecimento e manutenções, a tração requerida para cada trem e a capacidade de tração das locomotivas.

Em geral, o objetivo da programação é aumentar a utilização média das locomotivas e, consequentemente, reduzir o número total de máquinas necessárias para o transporte ferroviário. Contudo, a falta de ferramentas de planejamento e programação para suportar as decisões desta atividade resulta na utilização ineficiente das locomotivas, um dos recursos mais caros das ferrovias (MACHADO, 2006). Assim, realizou-se uma pesquisa bibliográfica com o objetivo de identificar os principais modelos matemáticos, propostos na literatura, que tratam do problema de alocação de locomotivas.

Portanto, para o desenvolvimento desta revisão bibliográfica, seguiu-se o procedimento proposto por Thomé et al. (2014). Assim, as buscas foram realizadas nas bases de dados Web of Science, Science Direct e Scopus, sem restrição do período, por meio da seguinte combinação de palavras-chave: '(locomotive and (assignment or distribuition or logistic* or allocation) and (problem or model* or optimization or algorithm)'.

Como resultado, foram identificados 151 artigos, que foram consolidados e avaliados da seguinte forma: análise do título e do resumo dos trabalhos; exclusão de artigos duplicados; e aplicação dos critérios de inclusão. Foram considerados apenas artigos com propostas de modelo matemáticos de otimização com foco no transporte ferroviário de cargas relacionados com a alocação de locomotivas e, preferencialmente, relacionados com timetable.

Assim, do total de 151 artigos, 49 foram eliminados por tratar do transporte de passageiros, 85 foram excluídos pois não abordavam o problema de alocação de locomotivas e 2 por tratarem de uma revisão bibliográfica sobre o tema, sem apresentar um modelo matemático. Dessa forma, 15 artigos foram selecionados e analisados neste trabalho, servindo como base para a revisão bibliográfica desenvolvida, conforme apresentado na tabela 1.

Os trabalhos selecionados foram analisados considerando as principais características presentes nos estudos sobre otimização na alocação de recursos. Elementos como horizonte e níveis de planejamento, além da função objetivo e tipos de modelagem, entre outros, foram utilizados para agrupar os estudos 
elaborados sob os mesmos conceitos e então facilitar a análise dos mesmos. Deste modo, a partir da análise dos artigos, algumas características puderam ser identificadas como sendo comuns em diversos trabalhos. A tabela 1 sintetiza este conjunto de artigos, apresentando suas principais características (problema abordado, método de resolução e função objetivo).

Tabela 1: Artigos selecionados para a revisão bibliográfica.

\begin{tabular}{|c|c|c|c|}
\hline Autor & Problema abordado & Método de resolução & Função objetivo \\
\hline Ziarati et al. (1997) & Alocação de locomotivas heterogêneas para o transporte de carga & $\begin{array}{l}\text { Exata - Decomposição de } \\
\text { Dantzig-Wolfe }\end{array}$ & $\begin{array}{l}\text { Min Custos Operacionais } \\
\text { Totais }\end{array}$ \\
\hline Ziarati et al. (2005) & $\begin{array}{c}\text { Otimização da alocação de locomotivas através da utilização de } \\
\text { inteligência artificial }\end{array}$ & Heurística & $\begin{array}{l}\text { Min Quantidade de } \\
\text { Locomotivas }\end{array}$ \\
\hline Ahuja et al. (2005) & Resolução do problema de alocação de locomotivas & Heurística & $\begin{array}{l}\text { Min Custos Operacionais } \\
\text { Totais }\end{array}$ \\
\hline Rouillon et al. (2006) & Aplicação do branch-and-bound para a alocação de locomotivas & Heurística & $\begin{array}{l}\text { Min Custos Operacionais } \\
\text { Totais } \\
\end{array}$ \\
\hline Kuo et al. (2007) & $\begin{array}{l}\text { Modelagem matemática para aumentar a utilização de locomotivas no } \\
\text { transporte de carga }\end{array}$ & Programação Inteira Mista & $\begin{array}{l}\text { Min Custos Operacionais } \\
\text { Totais } \\
\end{array}$ \\
\hline $\begin{array}{l}\text { Vaidyanathan et al. } \\
\text { (2008) }\end{array}$ & Problema de alocação de locomotivas & Programação Inteira Mista & $\begin{array}{l}\text { Min Custos Operacionais } \\
\text { Totais }\end{array}$ \\
\hline $\begin{array}{c}\text { Aronsson et al. } \\
(2010)\end{array}$ & Modelo de MIP para alocação e roteamento de locomotivas & Programação Inteira Mista & $\begin{array}{l}\text { Min Quantidade de } \\
\text { Locomotivas }\end{array}$ \\
\hline Ghoseiri et al. (2010) & Algoritmo genético para alocação de locomotivas com janelas de tempo & Heurística & $\begin{array}{l}\text { Min Custos Operacionais } \\
\text { Totais }\end{array}$ \\
\hline Su et al. (2015) & Otimização da alocação de locomotivas para o transporte de carga & Exata - Algoritmo Húngaro & $\begin{array}{l}\text { Min Quantidade de } \\
\text { Locomotivas }\end{array}$ \\
\hline $\begin{array}{c}\text { Teichmann et al. } \\
\text { (2015) }\end{array}$ & $\begin{array}{l}\text { Problema de alocação de uma frota heterogênea de locomotivas com } \\
\text { contratação de locomotivas externas }\end{array}$ & Exata - Programação Linear & $\begin{array}{l}\text { Min Custos Operacionais } \\
\text { Totais }\end{array}$ \\
\hline Zhang et al. (2015) & Modelo de alocação de locomotivas baseado em partição gráfica & Programação Inteira Mista & $\begin{array}{l}\text { Min Quantidade de } \\
\text { Locomotivas }\end{array}$ \\
\hline Azanov et al. (2016) & Modelo de alocação de locomotivas em trens de carga & $\begin{array}{l}\text { Heurística e Programação } \\
\text { Inteira }\end{array}$ & $\begin{array}{l}\text { Min Quantidade de } \\
\text { Locomotivas }\end{array}$ \\
\hline Ivanov et al. (2016) & $\begin{array}{l}\text { Modelo de otimização estocástica para alocação de locomotivas em trens } \\
\text { de carga }\end{array}$ & Heurística & $\begin{array}{l}\text { Máx Tempo de Utilização de } \\
\text { Locomotivas }\end{array}$ \\
\hline Jaumard et al. (2016) & Problema de alocação de locomotivas através da geração multicoluna & Exata - Decomposição & $\begin{array}{l}\text { Min Quantidade de } \\
\text { Locomotivas }\end{array}$ \\
\hline Xu et al. (2016) & Problema de alocação de locomotivas para a programação de trens & $\begin{array}{l}\text { Heurística e Programação } \\
\text { Inteira }\end{array}$ & Min do Atraso Total dos Trens \\
\hline
\end{tabular}

Em relação ao horizonte de planejamento para o qual os modelos foram desenvolvidos, autores como Rouillon et al. (2006) e Kuo et al. (2007) utilizam uma visão de programação semanal para a melhor alocação das locomotivas. Ahuja et al. (2005) considera um planejamento diário, com trens cíclicos que são formados pelo menos cinco vezes por semana. Jaumard et al. (2016), por sua vez, considera um horizonte de seis semanas na aplicação do modelo proposto na Canada Pacific Railway.

Os demais autores não definem um horizonte de planejamento. No que se refere ao nível de planejamento em que os modelos são desenvolvidos e em que sua aplicação é esperada, os autores classificam entre estratégico, tático ou operacional (ROUILLON et al., 2006; ZIARATTI et al., 1997). Em geral, atividades de programação de curto prazo e roteirização de locomotivas estão inseridas no nível operacional, enquanto o planejamento da alocação trata dos níveis estratégico e tático.

Ziarati et al. (1997), Vaidyanathan et al., (2008) e Azanov et al. (2016) têm uma abordagem de nível operacional, atuando na roteirização dos trens segundo os horários de circulação. Por sua vez, Ziarati et al. (2005), Ahuja et al. (2005), Aronsson et al. (2010) e Su et al. (2015) atuam em nível estratégico, na elaboração de um planejamento da alocação que considere tanto as necessidades dos clientes quanto os custos de utilização dos recursos do operador ferroviário.

A quantidade de diferentes tipos de locomotivas considerados no problema de alocação é um fator que tem influência tanto na elaboração do modelo quanto em seu desempenho, bem como restrições na 
associação de tipos específicos para determinados trens. Nesse sentido, Kuo et al. (2007), Ghoseiri et al. (2010) e Xu et al. (2016) trabalham com um único modelo de locomotiva que deve ter sua alocação otimizada. Por outro lado, Teichmann et al. (2015) e Zhang et al. (2015) consideram múltiplos modelos que podem ser alocados para tracionar os trens. Dos trabalhos analisados, Ahuja et al. (2005) e Su et al. (2015) propõem a blocagem de locomotivas de modo a simplificar a complexidade do modelo proposto. Jaumard et al. (2016) realiza um agrupamento dos diferentes tipos de trens que são atendidos por um mesmo modelo de locomotiva.

Em geral, os artigos analisados adotam programação inteira, heurística ou até a combinação de ambos para a modelagem do problema de alocação. Kuo et al. (2007), Vaidyanathan et al. (2008), Aronsson et al. (2010) e Zhang et al. (2015) recorrem à programação inteira na modelagem do problema de alocação. A aplicação de decomposição Dantzig-Wolfe, branch-and-bound e algoritmo genético são os principais métodos utilizados pelos autores dos demais trabalhos selecionados.

Em relação ao objetivo, a maioria dos modelos propostos busca a minimização: dos custos operacionais totais (ZIARATI et al., 1997; AHUJA et al., 2005; ROUILLON et al., 2006, KUO et al., 2007; VAIDYANATHAN et al., 2008; GHOSEIRI et al., 2010; TEICHMANN et al., 2015) ou da quantidade de locomotivas utilizadas no transporte (ZIARATI et al., 2005; ARONSSON et al., 2010; SU et al., 2015; ZHANG et al., 2015; AZANOV et al., 2016; JAUMARD et al., 2016). O modelo proposto por Ivanov et al. (2016) tem o objetivo de maximizar o tempo de utilização das locomotivas no transporte, enquanto Xu et al. (2016) busca a redução do tempo total de atraso na circulação de trens.

Por sua vez, a restrição mais adotada na modelagem do problema de alocação de locomotivas se refere ao fornecimento de tração de modo a atender a demanda de trens, estando presente em todos os trabalhos. Ainda, a capacidade tratora das locomotivas, a potência mínima necessária para deslocar o trem em sua rota específica, restrições relacionadas ao peso e comprimento dos trens, quantidades máximas ou mínimas de locomotivas ou de grupos tratores por trem e disponibilidade de locomotivas de cada modelo são as principais restrições presentes.

A partir destas análises, identificou-se o modelo proposto por Kuo et al. (2007) como aquele que mais se assemelha ao contexto estudado para elaboração deste trabalho. As características como movimentação de locomotivas entre pátios, horizonte de planejamento semanal e objetivo de redução de custos operacionais são aquelas que mais aproximam os dois estudos.

Contudo, de modo a tornar o modelo proposto mais representativo à realidade das empresas ferroviárias no Brasil, há a necessidade de inclusão de alguns fatores, como existência de múltiplos pátios ferroviários, tempos de circulação diferentes para rotas distintas e utilização de mais de um modelo de locomotiva para a realização do transporte. Além disto, buscou-se incluir na análise aspectos como a redução dos impactos ambientais da operação de transporte por meio de uma maior eficiência energética, cuja importância é discutida na Seção 3. A apresentação do modelo proposto é realizada na Seção 4. 


\section{Eficiência energética e impactos ambientais}

Salum (2005) define eficiência energética como o uso da energia de forma a obter o máximo benefício com o menor consumo, evitando desperdícios ou o uso inadequado, sem diminuir a qualidade de vida ou produtividade. Do ponto de vista do setor ferroviário, Pereira (2009) define a eficiência energética como a razão do consumo de combustível de uma locomotiva em relação à quantidade de carga tracionada por uma determinada distância, de modo que uma locomotiva apresente bom resultado em eficiência energética caso economize combustível e/ou aumente a carga bruta tracionada do trem.

O aprimoramento do uso de energia e da gestão da eficiência energética vem sendo necessário não só em relação ao contexto econômico, como resposta aos aumentos dos preços, mas ganha cada vez mais importância na mitigação aos impactos ambientais às mudanças climáticas. Para Aragón et al. (2013), a eficiência energética surge como uma alternativa na busca da redução da poluição ambiental, além de melhorar tanto a produtividade quanto a competitividade das empresas a partir da diminuição do consumo de energia e, consequentemente, redução dos custos operacionais.

As emissões brasileiras de dióxido de carbono $\left(\mathrm{CO}_{2}\right)$ vêm aumentando gradativamente e estão diretamente relacionadas à queima de combustíveis fósseis (ARAÚJO, 2006; D’AGOSTO, 2015). Entre todos os setores que emitem $\mathrm{CO}_{2}$, o setor de transportes apresenta a maior participação quanto ao total de emissões, representando, em 2013, 46\% e 23\% das emissões de fontes de combustíveis fósseis no Brasil e no Mundo, respectivamente (IEMA, 2016). Por sua vez, o transporte ferroviário representa cerca de $5 \%$ do total de $\mathrm{CO}_{2}$ emitido pelo transporte terrestre de cargas no Brasil.

Em termos de evolução das emissões, as ferrovias brasileiras aumentaram em, aproximadamente, $45 \%$ a quantidade de $\mathrm{CO}_{2}$ emitida entre 2002 e 2011, saindo de um patamar de 2.125 para 3.079 mil toneladas (ANTT, 2012). Deste modo, o aumento da eficiência energética no setor de transportes tem impacto direto nas emissões de GEE.

A principal fonte de energia do transporte ferroviário de cargas no Brasil é o óleo diesel, sendo que a maior parte das locomotivas no país possuem motor diesel-elétrico (ANTT, 2012). Todavia, o transporte ferroviário destaca-se por sua maior eficiência energética e, consequentemente, uma menor emissão de GEE por unidade transportada quando comparado ao modo rodoviário. De tal modo, pode-se afirmar que o incentivo ao transporte ferroviário de cargas contribui para a redução das emissões de GEE e, de fato, este é um tema que tem ganhado destaque no cenário nacional.

Em 2012, por exemplo, foi elaborado o 10 Inventário Nacional de Emissões Atmosféricas do Transporte Ferroviário de Cargas, que aborda questões ambientais e econômicas, como emissão de gases de efeito estufa e consumo de combustíveis, dentre outros aspectos, disponibilizando informações acumuladas desde o início da atuação da Agência Nacional de Transportes Terrestres (ANTT, 2012).

É neste contexto que a gestão dos indicadores de eficiência energética nas ferrovias torna-se importante, na medida em que se conhecem as variáveis que influenciam este processo, e sua importância estratégica para o aumento da produtividade, otimização de custos e redução de impactos ambientais 
negativos. Dado que uma alocação eficiente de locomotivas pode contribuir com, pelo menos, cerca de $5 \%$ para a redução do consumo energético (CASSEMIRO et al., 2015), tendo assim influência direta no aumento da eficiência energética e na redução das emissões de GEE, este problema ganha uma relevância ainda maior, principalmente quando se considera também a questão econômica, pois esta é a segunda maior parcela na matriz de custos de uma empresa ferroviária (AAR, 2018).

\section{MATERIAIS E MÉTODOS}

\section{Procedimento para a alocação das locomotivas}

O problema de alocação de locomotivas possui uma série de abordagens de resolução apresentada nas diversas publicações analisadas na revisão sistemática da literatura (Seção 2). Fatores como horizonte de planejamento, níveis de planejamento e tipos de locomotivas são alguns dos que influenciam e determinam como será a aplicação dos modelos propostos.

Tomando como base estes fatores e o contexto de aplicação deste trabalho, a proposta é realizar a alocação em nível operacional, com a programação das locomotivas em cada um dos trens selecionados. Essa abordagem se torna relevante no contexto operacional da ferrovia, uma vez que permite um melhor aproveitamento das oportunidades na programação dos ativos, além da melhor alocação dos recursos disponíveis de acordo com a demanda de trens. O procedimento proposto é composto por seis etapas: seleção dos trens; identificação das locomotivas disponíveis nos pátios; cálculo do custo de manutenção das locomotivas; cálculo do custo de combustível; alocação das locomotivas nos trens; e cálculo da emissão de $\mathrm{CO}_{2}$.

Na primeira etapa do procedimento, o objetivo é identificar quais os trens serão selecionados para a realização do estudo de alocação das locomotivas. Assim, considera-se uma ferrovia de transporte de carga, que possui trens com grade horária, a qual devem seguir rigorosamente, parando em diversos pátios e terminais ao longo de sua rota para anexar e retirar vagões, sendo que as paradas têm horários definidos de chegada e partida.

Apesar de estes trens serem formados por vagões de diferentes clientes, o que implica em os vagões terem destinos diferentes, o trem possui uma rota fixa, constituída por uma origem e um destino, que nem sempre coincide com a rota de todos os vagões que estão presentes na composição. Dessa forma, as locomotivas tendem a circular respeitando a rota do trem, e não dos vagões, ou seja, estas circulam desde a origem até o destino do trem.

A substituição de uma locomotiva só acontece quando há a necessidade de manutenção ou troca por avaria, e mesmo assim, por outro veículo idêntico. Ainda em função do volume a ser transportado e dos terminais a serem atendidos, existem trens que possuem a mesma origem de circulação, mas com destinos diferentes ao longo da malha ferroviária. Isso significa que de um mesmo pátio pode sair mais de um trem no mesmo dia, transportando produtos para diversos clientes. Esses fatos geram uma necessidade de decisão por parte da programação dos trens de qual locomotiva alocar em cada uma das composições que circulam 
entre um pátio de origem e um pátio de destino, uma vez que, em geral, existe mais de um modelo disponível e mais de um trem que requer as locomotivas.

A segunda etapa consiste em identificar quais locomotivas estarão disponíveis em cada um dos pátios. Esses pátios coincidem com as origens dos trens selecionados, de onde as locomotivas iniciam sua circulação. A disponibilidade da locomotiva no pátio representa que a máquina está ou estará no pátio antes da partida do trem em boas condições, ou seja, não se encontra avariada, e não está destinada a um outro atendimento.

Em um contexto de programação semanal, é importante ressaltar que as locomotivas tendem a ser utilizadas mais de uma vez e em mais de um trem, desde que o tempo de ciclo entre a origem e o destino seja inferior a 7 dias, e estarão disponíveis em diferentes pátios ao longo do horizonte de planejamento. Isto quer dizer que uma locomotiva voltará a estar disponível para um segundo trem no pátio de destino do primeiro trem no qual esta circulou, em um período que é igual ao tempo de ciclo do primeiro trem, por exemplo.

$\mathrm{Na}$ terceira etapa do procedimento, são determinados os custos de manutenção associados à alocação de um modelo de locomotiva em um trem. As revisões e inspeções acontecem periodicamente nas locomotivas e, em geral, a necessidade de manutenção se dá pela quilometragem total percorrida, pelas horas disponíveis para utilização ou pela quantidade de óleo diesel consumido no período.

Dessa forma, fatores como tempo de viagem e extensão das rotas percorridas pelos trens, além da disponibilidade e utilização da frota afetam diretamente na periodicidade em que as locomotivas vão as oficinas, realizam troca de peças e utilizam o homem-hora da manutenção. Sendo assim, dependendo de para qual trem a locomotiva será alocada, o custo deste será maior ou menor, visto que os diferentes modelos de locomotivas possuem diferentes custos de manutenção associados. Portanto, a decisão de direcionar locomotivas de baixo custo para trens onde os gatilhos de manutenção serão acelerados por ineficiências como altos tempos de viagem, alto consumo de óleo diesel ou grandes distâncias percorridas tornam-se estratégica do ponto de vista dos custos do transporte.

Para este trabalho, propõe-se a determinação de um custo médio de manutenção (C_Manut), composto pela razão entre o somatório de todos os custos, dado o fechamento de um ciclo de manutenção de uma determinada locomotiva, e o momento de manutenção deste ativo, que neste caso será a tonelada bruta transportada pelo trem vezes distância percorrida (TKB) neste período.

Por exemplo, um determinado modelo possui manutenções disparadas por uma determinada TKB transportada e inspeções quinzenais, revisões trimestrais, semestrais, anuais e revisão geral bianual, fechando assim um ciclo de manutenção em dois anos. Desta forma o C_Manut é dado pelo valor gasto nestes dois anos com peças e serviços dividido por toda a TKB transportada pela locomotiva neste mesmo período. Como cada trem possui uma rota e um peso, é possível determinar o custo de manutenção, em R\$S/TKB, associado à locomotiva alocada ao trem. Propõe-se: $C_{-}$Manut $=$ $\frac{\text { Custo total de manutenção durante o ciclo }}{\text { TKB total tracionado duranto o ciclo }}$ 
Na quarta etapa, calcula-se o custo com combustível resultante da alocação de um determinado modelo de locomotiva a um trem. Modelos diferentes de locomotivas vão apresentar diferentes eficiências energéticas e, por isso, podem se tornar mais ou menos interessantes para determinados trens. Assim, esse custo se torna determinante na otimização da alocação de locomotivas em trens.

Analisando os diferentes modelos de locomotivas, bem como os diferentes trens, em termos de rota, distância percorrida e volume transportado, que circulam na malha ferroviária, tem-se a estimativa da

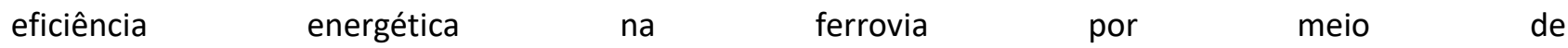
$E E=\frac{L}{T k b} \cdot 10^{-3}=\left(\frac{L_{1}}{T k b_{1}}+\frac{L_{2}}{T k b_{2}}+\cdots+\frac{L_{n}}{T k b_{n}}\right) \cdot 10^{-3}$, em que EE: eficiência energética [L.10-3/TKB]; L: litros de diesel consumidos [L]; TKB: distância percorrida x tonelada bruta transportada [TBxKm]; e n: total de tipos de trens que circulam na malha ferroviária

Deste modo, para se obter o custo do diesel associado à alocação, é necessário estimar a quantidade de litros consumidos pelo modelo de locomotiva quando alocada a um trem que percorre determinada rota: $L_{i j k}=E E_{i j k} \cdot T k b_{i j} \cdot 10^{-3}$, em que EE: eficiência energética [L.10-3/TKB]; L: litros de diesel consumidos [L]; TKB: distância percorrida $x$ tonelada bruta transportada [TBxKm]; i: pátio de origem de trens que circulam na malha ferroviária; j: pátio de destino de trens que circulam na malha ferroviária; e k: modelo de locomotiva.

A partir da segunda equação, é possível elencar diversos fatores diretos e indiretos que impactam no resultado da eficiência energética. Alguns indiretos são: características geométricas da rede ferroviária, forma de condução da locomotiva, licenciamento para circulação de trens e característica dos veículos ferroviários. Já os fatores diretos correspondem à distância percorrida e ao volume transportado pela composição (CASSEMIRO et al., 2015).

Uma vez que são utilizados dados históricos de circulação dos trens para a aplicação do modelo proposto, ambos os tipos de fatores se tornam presentes quando apurada a eficiência energética dos trens considerados, já que estes circulam pelo mesmo trajeto, com peso conhecido e com padrões de condução e circulação preestabelecidos.

Na quinta etapa do procedimento, realiza-se a alocação dos diversos modelos de locomotivas a cada tipo de trem que circula na malha ferroviária. A partir da revisão bibliográfica realizada para este trabalho sobre alocação de locomotivas, verificaram-se tanto os métodos de resolução quanto as funções objetivo e restrições aplicadas pelos diversos autores nos modelos propostos. Dessa forma, optou-se pela resolução do problema de alocação de locomotivas através do uso de programação linear inteira, uma vez que este problema pode ser resolvido com baixo esforço computacional. Além disso, essa forma de resolução se apresentou como sendo o principal método utilizado na literatura estudada. A tabela 2 apresenta a notação matemática utilizada neste trabalho.

Tabela 2: Notação matemática utilizada.

\begin{tabular}{|c|c|l|}
\hline Tipo & Notação & \\
\hline Conjunto & $T$ & conjunto dos dias da semana (índice $t$ ) \\
\hline Conjunto & $M$ & conjunto de pátios considerão \\
\hline Conjunto & $N$ & conjunto de modelos de locomotivas considerado no modelo de alocação (índices $i$ e $j$ ) \\
\hline $\begin{array}{c}\text { Variável de } \\
\text { decisão }\end{array}$ & $X_{i j k t}$ & $\begin{array}{l}\text { representa a alocação para o trem que circula do pátio } i \text { para o pátio } j \text { com o modelo de locomotiva } k \text { no dia } t \text { da } \\
\text { semana de programação }\end{array}$ \\
\hline
\end{tabular}




\begin{tabular}{|c|c|c|}
\hline $\begin{array}{c}\text { Variável de } \\
\text { decisão }\end{array}$ & Disp_Loco $i k t$ & representa a quantidade disponível no pátio $i$ do modelo de locomotivas $k$ no dia $t$ da semana de programação \\
\hline $\begin{array}{c}\text { Variável de } \\
\text { decisão }\end{array}$ & $S_{k}$ & representa o tamanho da frota do modelo de locomotivas $k$ \\
\hline $\begin{array}{c}\text { Variável de } \\
\text { decisão }\end{array}$ & Chegada $_{i k t}$ & representa a quantidade que chega no pátio $i$ do modelo de locomotivas $k$ no dia $t$ da semana de programação \\
\hline Parâmetro & Preço_Litro & preço do litro do óleo diesel \\
\hline Parâmetro & C_Manut M $_{\text {- }}$ & custo histórico de manutenção do modelo de locomotiva $k$ \\
\hline Parâmetro & $L_{i j k}$ & litros de óleo diesel consumidos pelo modelo de locomotiva $k$ quando circula do pátio $i$ para o pátio $j$ \\
\hline Parâmetro & $T K B_{i j}$ & distância percorrida $x$ tonelada bruta transportada pelo trem quando circula do pátio $i$ para o pátio $j$ \\
\hline Parâmetro & $\mathrm{TC}_{\mathrm{ji}}$ & tempo de ciclo entre o pátio $j$ e o pátio $i$ \\
\hline Parâmetro & Demanda $_{i j t}$ & demanda de circulação de trens do pátio $i$ para o pátio $j$ no dia $t$ da semana de programação \\
\hline Parâmetro & $G_{i k t}$ & locomotivas que chegam no pátio $i$ do modelo $k$ no dia $t$ da semana de programação \\
\hline
\end{tabular}

Desta forma, com o objetivo de otimizar a alocação das locomotivas para um conjunto de trens em um horizonte de programação semanal, de modo a minimizar o custo do transporte em termos de gastos com combustível e manutenção e assim considerando a eficiência energética da operação, é proposto o modelo representado pelas expressões que seguem.

Minimizar $\sum_{t=1}^{T} \sum_{i=1}^{M} \sum_{j=1}^{M} \sum_{k=1}^{N}\left(C_{-}\right.$Manut $_{k} . T K B_{i j}+L_{i j k}$.Preço_Litro $) . X_{i j k t}, \quad$ sujeito $\quad$ a: $j \neq i$

$\sum_{k=1}^{N} X_{i j k t} \geq$ Demanda $_{i j t}, \forall t \in T, \forall i \in M, \forall j \in M, i \neq j ; \sum_{i=1}^{M} \sum_{j=1}^{M} X_{i j k t} \leq \sum_{i=1}^{M}$ Disp_Loco $_{i k t}, \forall t \in$ $j \neq i$

$T, \forall k \in N ; \quad \sum_{i=1}^{M}$ Disp_Loco $_{i k t} \leq S_{k}, \quad \forall t \in T, \forall k \in N ; \quad$ Disp_Loco $_{i k t}+$ Chegada $_{i k t}-\sum_{j=1}^{M} X_{i j k t}=$ $j \neq i$

Disp_Loco $_{i k t+1}, \quad \forall t \in T, \forall i \in M, \forall k \in N ; \quad$ Chegada $_{i k t}=G_{i k t}, \quad \forall t \in T$ e $t \leq t c_{j i}, \forall i \in M, \forall k \in N$; Chegada $_{i k t}=\sum_{j=1}^{M} X_{j i k\left(t-t c_{j i}\right)}, \forall t \in T$ e $t>t c_{j i}, \forall i \in M, \forall k \in N ; X_{i j k t} \geq 0$ e inteiro, $\forall i \in M, \forall j \in$ $j \neq i$

$M, \forall k \in N, \forall t \in T ;$ Disp_Loco $_{i k t} \geq 0$ e inteiro, $\forall i \in M, \forall k \in N, \forall t \in T$; e Chegada ${ }_{i k t} \geq 0$ e inteiro, $\forall i \in M, \forall k \in N, \forall t \in T$.

A função objetivo apresenta a minimização dos custos de manutenção e combustível, considerando o custo médio de manutenção de cada modelo de locomotiva $k$ (C_Manut ${ }_{k}$ ), em R\$\$/TKB, multiplicado pela distância percorrida $x$ tonelada bruta $\left(T_{K} B_{i j}\right)$ do trem que circula do pátio de origem $i$ para o pátio de destino $j$, sendo que $i$ é diferente de $j$, além dos litros de diesel consumidos ( $\left.L_{i j k}\right)$ pelo trem que circula do pátio de origem $i$ para o pátio de destino $j$ com o modelo de locomotiva $k$, em $\mathrm{R} \$$ /litro, vezes o preço do litro. Somados os custos, este é contabilizado caso seja feita a alocação do modelo de locomotiva $k$, no trem que circula de $i$ para $j$ no dia $t$ da semana.

Por sua vez, as restrições garantem que o somatório da alocação de todos os modelos de locomotivas $k$ para o trem que circula de $i$ para $j$ no dia $t$ da semana deve ser maior ou igual à demanda de trens que circulam de $i$ para $j$ no dia $t$ da semana; as restrições garantem que o somatório da alocação do modelo de locomotivas $k$ para os trens que circula entre o pátio de origem $i$ para todos os pátios de destino $j$ no dia $t$ da semana deve ser menor ou igual à disponibilidade no pátio de origem $i$ do modelo de locomotivas $k$ no dia $t$ da semana.

As restrições garantem que a disponibilidade no pátio de origem $i$ do modelo de locomotivas $k$ no dia $t$ da semana está restrita ao tamanho da frota de locomotivas $\mathrm{S}$ de cada modelo; as restrições garantem que a disponibilidade no pátio de origem $i$ do modelo de locomotivas $k$ no dia $t$ da semana mais o que está 
chegando em $i$ do modelo de locomotivas $k$ no dia $t$ menos o somatório do que está sendo alocado do pátio de origem $i$ para todos os pátios de destino $j$ com o modelo de locomotivas $k$ no dia $t$ da semana será igual à disponibilidade no pátio de origem $i$ do modelo de locomotivas $k$ no dia $t+1$ da semana.

As restrições garantem que a chegada de locomotivas no pátio $i$ do modelo $k$ no dia $t$ será igual ao parâmetro $G_{i k t}$, quando $t$ for menor ou igual ao tempo de ciclo entre $j$ e $i$; as restrições garantem que a chegada de locomotivas no pátio $i$ do modelo $k$ no dia $t$ será igual ao somatório do que foi alocado dos pátios $j$ para o pátio $i$ com o modelo de locomotivas $k$ no dia da semana $t$ menos o tempo de ciclo entre $j$ e $i$, quando $t$ for maior que o tempo de ciclo entre $j$ e $i$; as últimas restrições garantem a positividade e integralidade das variáveis de decisão.

Por fim, na sexta etapa, estima-se a redução de emissão de $\mathrm{CO}_{2}$ obtida por meio de uma alocação mais eficiente das locomotivas. Assim, sugere-se a adoção da metodologia proposta pela Environmental Protection Agency (EPA, 2009). Esta metodologia permite o cálculo da emissão de $\mathrm{CO}_{2}$ por litro de diesel consumido por locomotivas por meio do produto entre a densidade do diesel $(\mathrm{kg} / \mathrm{l})$, o percentual de presença do Carbono no Diesel (\%) e o percentual de presença do $\mathrm{CO}_{2}$ na molécula de Carbono (\%). Deste modo, é possível realizar a comparação entre o cenário real de alocação de locomotivas com a alocação otimizada resultante do modelo.

\section{Aplicação do procedimento proposto}

Esta seção apresenta os critérios utilizados para a aplicação de cada uma das etapas do procedimento apresentado. De modo a verificar a aplicabilidade e consistência do procedimento proposto, realizou-se a aplicação a um caso de uma empresa de transporte ferroviário de cargas brasileira, a qual é atualmente responsável por um terço de toda a carga transportada por trens no país. As principais cargas transportadas pela empresa são commodities minerais e agrícolas, além de contêineres, siderúrgicos, cimento, bauxita, entre outros produtos. A figura 1 apresenta um mapa com a região de atuação da empresa analisada, com a sinalização dos pátios que são origens/destinos dos trens considerados.

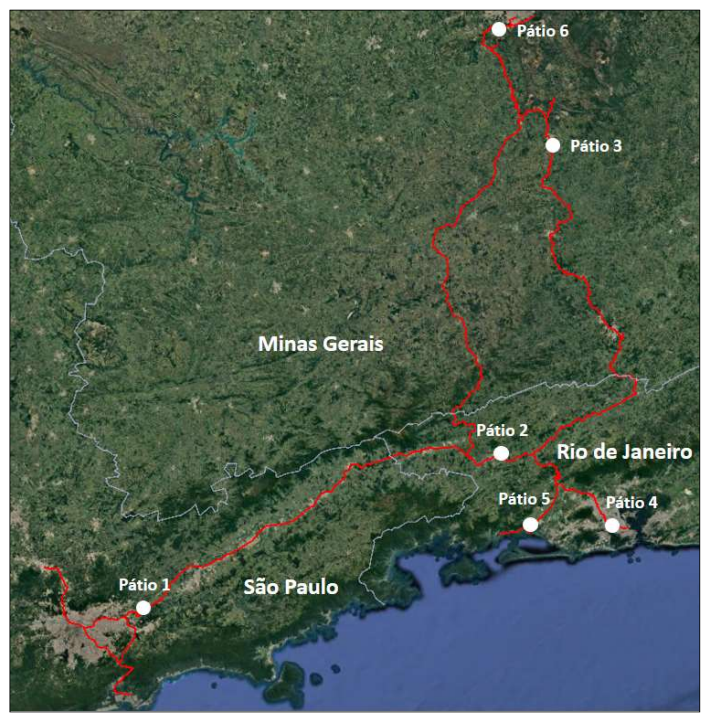

Figura 1: Região de atuação da empresa ferroviária estudada. 
Inicialmente, foi feita a seleção dos trens a serem considerados na otimização da alocação das locomotivas. Foram analisadas as rotas de trens entre os pátios considerados e que possuíam horários predeterminados de circulação frente ao total de trens existentes na malha ferroviária. Onze tipos de trens foram considerados no modelo, com origens e destinos entre os pátios selecionados, representando cerca de $85 \%$ do volume transportado por trens que possuem grade horária de circulação. Cada um dos trens selecionados possui demanda de vagões para circular até uma vez por dia e necessita de uma locomotiva para fornecimento de tração. Sendo assim, para o horizonte semanal considerado, a demanda máxima é de 77 trens. Para o cenário analisado, a demanda de trens a ser atendida pelas locomotivas foi de 69 trens na semana.

Para a identificação das locomotivas disponíveis, foram considerados os 6 pátios que são origens e/ou destinos dos onze trens selecionados previamente. Esses pátios são os locais em que as locomotivas precisam estar disponíveis para que sejam alocadas nas composições. Em seguida, dado os diferentes tipos de locomotivas existentes, foram selecionados os 3 grupos $(10,9$ e 8) que são utilizados para tracionar os trens de produção em função de sua maior capacidade tratora e maior confiabilidade. Existem outros grupos (do 1 ao 7) disponíveis, porém que são utilizados apenas para manobras de pátios, trens de via, entre outras atividades. Chegou-se a um total de 49 locomotivas disponíveis para atendimento da demanda no início de horizonte de programação semanal. A tabela 3 apresenta essa distribuição no primeiro dia da semana.

Tabela 3: Distribuição inicial da quantidade de locomotivas disponíveis por grupo e pátio.

\begin{tabular}{|c|c|c|c|}
\hline \multirow{2}{*}{ Pátios } & \multicolumn{3}{|c|}{ Locomotivas } \\
\cline { 2 - 4 } & Grupo 10 & Grupo 9 & Grupo 8 \\
\hline Pátio 1 & 3 & 0 & 2 \\
\hline Pátio 2 & 3 & 6 & 0 \\
\hline Pátio 3 & 8 & 0 & \\
\hline Pátio 4 & 6 & 2 & \\
\hline Pátio 5 & 2 & 5 & \\
\hline Pátio 6 & 3 & & \\
\hline
\end{tabular}

Vale ressaltar que à medida que as locomotivas são alocadas aos trens, elas voltaram a estar disponíveis em outros pátios e num horizonte de tempo igual ao ciclo do trem em que foram alocadas anteriormente. Esse fato permite que algumas locomotivas sejam utilizadas em mais de um trem durante a semana de programação.

Para o cálculo do custo de manutenção, realizou-se o levantamento do custo total dado o fechamento do ciclo de manutenção de cada locomotiva nos anos de 2016 e 2017. Além disso, levantou-se o momento de transporte (TKB) tracionado pelas locomotivas no mesmo período. Assim, estimou-se o Custo/TKB, em R\$̦, que cada grupo de locomotiva possui. Relacionando essa informação como a TKB de cada um dos onze trens selecionados, foi possível determinar o custo de manutenção associado à locomotiva alocada ao trem que percorre determinada rota.

Por sua vez, para o cálculo do custo com combustível, primeiramente foi levantada a eficiência energética média histórica de cada grupo de locomotiva em cada um dos onze trens selecionados. De posse da informação e considerando o TKB de cada um dos trens que circularam na semana, foi possível estimar o consumo de combustível, em litros, e transformá-lo em custo por meio da multiplicação pelo preço por litro 
considerado, que foi $\mathrm{R} \$ 3,00$. A tabela 4 apresenta o resumo dos dois custos considerados para cada trem e para cada um dos grupos de locomotivas selecionados.

Tabela 4: Resumo dos custos de manutenção e combustível por trem e grupo de locomotiva (em R\$).

\begin{tabular}{|c|c|c|c|c|c|c|}
\hline \multirow{2}{*}{ Trens } & \multicolumn{3}{|c|}{ Custo de Manutenção } & \multicolumn{3}{|c|}{ Custo de Combustível } \\
\hline & Grupo 10 & Grupo 9 & Grupo 8 & Grupo 10 & Grupo 9 & Grupo 8 \\
\hline Trem 1 & 764,28 & $1.885,07$ & $6.728,84$ & $9.744,09$ & $11.620,88$ & $15.591,15$ \\
\hline Trem 2 & $2.223,35$ & $5.483,84$ & $19.574,84$ & $30.223,02$ & $34.948,20$ & $53.074,51$ \\
\hline Trem 3 & $1.089,20$ & $2.686,48$ & $9.589,53$ & $14.543,05$ & $17.215,98$ & $28.767,52$ \\
\hline Trem 4 & 976,35 & $2.408,14$ & $8.595,98$ & $13.845,74$ & $16.163,18$ & $26.334,21$ \\
\hline Trem 5 & 825,83 & $2.036,88$ & $7.270,74$ & $12.531,16$ & $13.619,99$ & $18.688,67$ \\
\hline Trem 6 & $1.302,93$ & $3.213,65$ & $11.471,28$ & $18.234,59$ & $21.597,99$ & $31.839,21$ \\
\hline Trem 7 & 518,18 & $1.278,07$ & $4.562,15$ & $6.996,23$ & $8.731,21$ & $12.950,86$ \\
\hline Trem 8 & 620,02 & $1.529,27$ & $5.458,79$ & $8.174,13$ & $9.481,29$ & $17.509,54$ \\
\hline Trem 9 & 637,97 & $1.573,54$ & $5.616,82$ & $8.761,63$ & $10.453,93$ & $17.100,89$ \\
\hline Trem 10 & 110,69 & 273,01 & 974,53 & $1.582,95$ & $1.756,40$ & $4.716,44$ \\
\hline Trem 11 & 355,79 & 877,54 & $3.132,42$ & $4.481,21$ & $5.552,60$ & $9.488,24$ \\
\hline
\end{tabular}

Com o valor dos parâmetros pré-calculados, realizou-se então a alocação das diversas locomotivas a cada tipo de trem que circula na malha ferroviária. O modelo apresentado na Seção 4 foi implementado no software LINGO 17.0, com o apoio do MS EXCEL para input/output dos dados. O ponto de partida do modelo foi a alocação realizada manualmente pelos usuários da empresa para uma semana típica do transporte entre os dias 5 e 11 de março de 2018. Utilizando os dados de densidade do combustível, além dos percentuais de $\mathrm{CO}_{2}$ no carbono e carbono no combustível para o tipo de óleo diesel utilizado pelas locomotivas da empresa, foi possível estimar o fator de emissão de $2,7211 \mathrm{kgCO}_{2} / \mathrm{l}$ de óleo diesel consumido. Multiplicando este fator pela quantidade de litros de óleo diesel que deixou de ser consumido por meio da alocação mais eficiente das locomotivas, foi possível realizar a comparação entre as emissões de $\mathrm{CO}_{2}$ nos cenários real e otimizado.

\section{RESULTADOS E DISCUSSÃO}

A aplicação do modelo proposto resultou na solução ótima, em termos de redução de custos e de emissão de $\mathrm{CO}_{2}$, para o problema de alocação de locomotivas apresentado. Como esperado, das 49 locomotivas disponíveis para utilização, algumas foram utilizadas mais de uma vez enquanto outras sequer foram necessárias para o transporte. Essa mesma situação aconteceu no cenário real, no qual 37 locomotivas foram necessárias para transporte, ou seja, 12 locomotivas não foram demandadas e poderiam ter sido utilizadas para outras finalidades. No cenário otimizado, o total de locomotivas não utilizadas subiu para 25 , representando uma redução na utilização das locomotivas de 35\% em relação à alocação real. A figura 2 apresenta a comparação de uso das locomotivas entre o cenário real e otimizado para o período de análise.

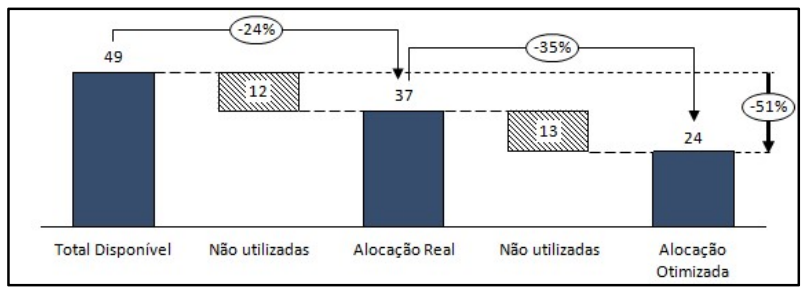

Figura 2: Comparação da utilização das locomotivas nos cenários. 
Com relação aos custos do transporte, o modelo proposto apresentou uma redução de $\mathrm{R} \$ 101.745,00$ por semana, correspondente a 10\%, em relação ao modelo de alocação adotado pela empresa, o qual não considera os custos de combustível e manutenção das locomotivas como um fator de decisão. Em termos anuais, essa economia chegaria a aproximadamente $\mathrm{R} \$ 5,3 \mathrm{mi}$. A figura 3 apresenta a comparação dos custos entre os cenários real e otimizado.

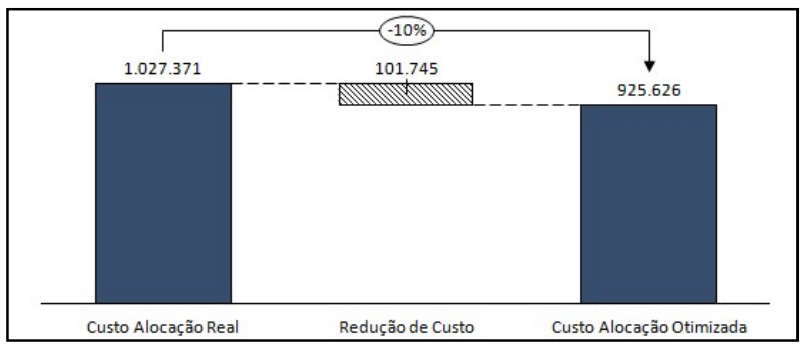

Figura 3: Comparação entre os custos de alocação no cenário real e o otimizado (em $R \$$ ).

Em relação ao consumo total de combustível, obteve-se uma redução de 20.537 litros no horizonte semanal, o que corresponde a 7\%, em relação ao modelo de alocação atual. Em termos anuais, essa economia seria de aproximadamente 1,1 milhão de litros. A figura 4 apresenta a comparação do consumo de combustível entre os cenários real e otimizado.

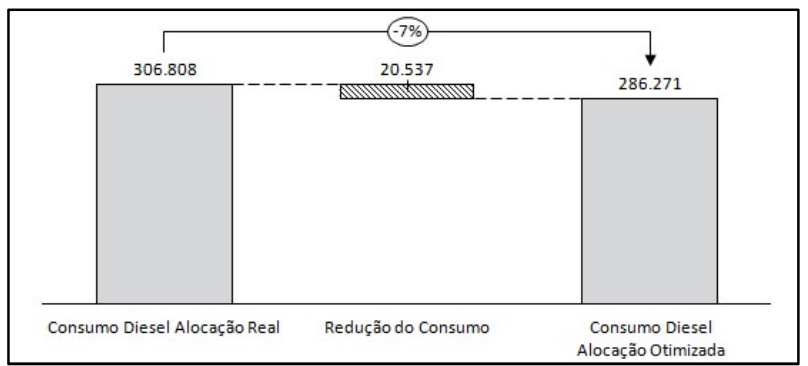

Figura 4: Comparação entre o consumo de diesel no cenário real e o otimizado (em litros).

Essa redução no consumo de combustível, quando convertido para emissão de $\mathrm{CO}_{2}$, apresenta uma redução de 56 toneladas do gás que deixariam de serem emitidos pela alocação eficiente de locomotivas. A figura 5 apresenta a comparação da emissão de $\mathrm{CO}_{2}$ entre os cenários real e otimizado. Em termos práticos, essa quantidade do gás que deixa de ser emitida é o equivalente ao plantio de aproximadamente 343 árvores, conforme dados do estudo realizado pela USP.

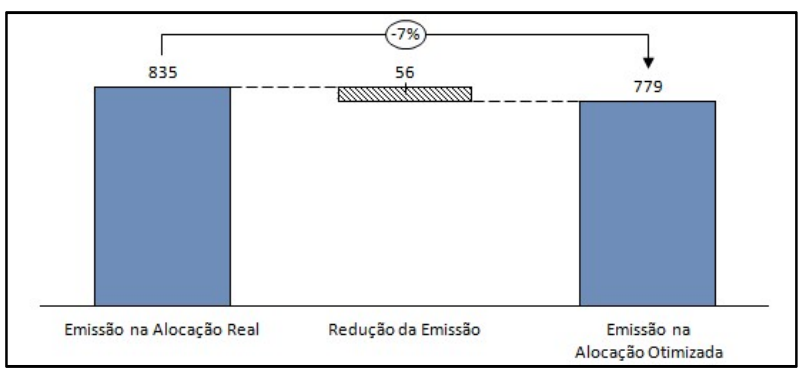

Figura 5: Comparação entre a emissão de $\mathrm{CO}_{2}$ no cenário real e o otimizado (em toneladas).

Ainda, de modo a avaliar os fatores que impactam nos resultados do processo de alocação de locomotivas, foram feitas análises de sensibilidade de alguns dos parâmetros do modelo. Inicialmente, 
optou-se por incrementar os custos de combustível com reajustes entre $10 \%$ e $50 \%$ enquanto os custos de manutenção se mantinham inalterados. Em seguida, realizou-se o processo contrário, ou seja, incrementar os custos de manutenção com reajustes entre $10 \%$ e $50 \%$ enquanto os custos de combustível se mantinham inalterados. O objetivo dessa análise consiste em verificar quais dos custos considerados tem maior impactos no custo total do transporte. As Figuras 6 e 7 apresentam os resultados obtidos.

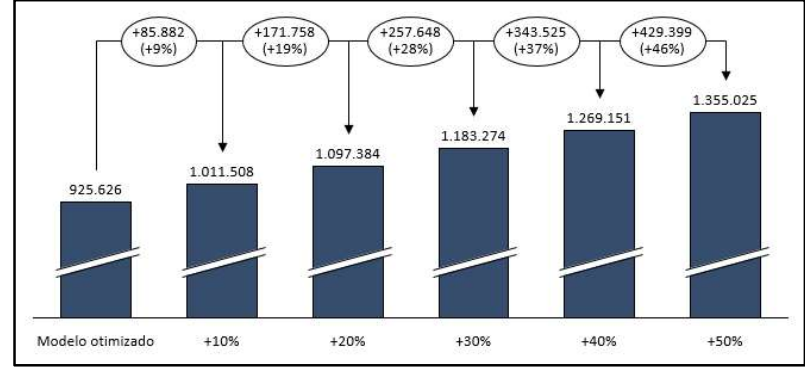

Figura 6: Variação do custo total do transporte em função de variações no custo de combustível (em $R \$$ ).

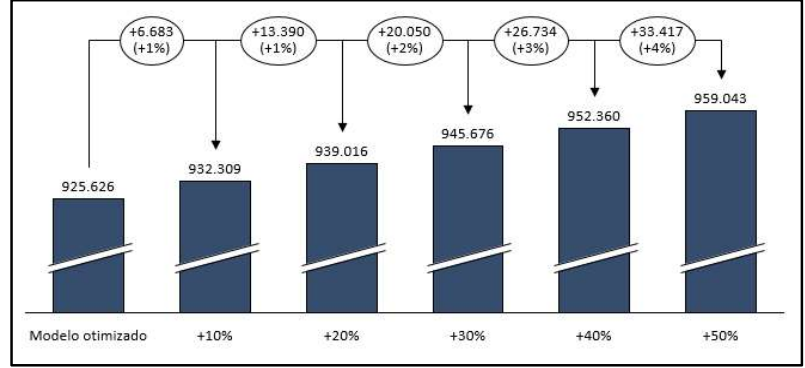

Figura 7: Variação do custo total do transporte em função de variações no custo de manutenção (em R\$).

Observa-se, por meio das figuras 6 e 7, que o custo de combustível é o fator que mais impacta no resultado global do custo do transporte. Um reajuste de $10 \%$ neste custo impacta em $9 \%$ no custo do transporte, por exemplo. Dessa forma, ações que estejam relacionadas com a redução dos custos de combustível e melhorias da eficiência energética das locomotivas devem ser priorizadas quando se objetiva a redução dos custos totais do transporte.

Em seguida, foi realizado um ajuste na disponibilidade inicial utilizada no modelo de alocação. Com a análise dos resultados, verifica-se que em alguns pátios faltaram locomotivas do grupo 10 (mais eficientes), ou seja, estas não estavam em quantidades suficientes para atender a todas as demandas. Com isso, locomotivas dos grupos 9 e 8, menos eficientes, foram utilizadas para o atendimento à demanda de trens. Dessa forma, foi feita a transferência de locomotivas do grupo 10 de pátios em que sobraram para pátios em que faltaram, para que estas pudessem ser alocadas aos trens. A tabela 5 apresenta a comparação dos resultados da otimização original com a otimização feita a partir do ajuste da disponibilidade inicial.

Tabela 5: Comparação dos resultados das otimizações realizadas com a disponibilidade original e com a disponibilidade ajustada.

\begin{tabular}{|l|c|c|c|}
\hline & Disponibilidade Original & Disponibilidade Ajustada & 23 \\
\hline Locomotivas Utilizadas & 24 & 911.153 & 1 \\
\hline Custo do Transporte (R\$) & 925.626 & 283.067 & 770 \\
\hline Litros Consumidos (L) & 286.271 & 3.473 \\
\hline Emissão de $\mathrm{CO}_{2}$ (ton) & 779 & 9 & 396 \\
\hline Ganho de Árvores & 343 & 53 & \\
\hline
\end{tabular}

A tabela 5 apresenta os ganhos adicionais em todos os itens considerados no processo de alocação com o ajuste da disponibilidade inicial. Contudo, vale observar que esse ajuste depende de movimentar as locomotivas entre os pátios que são as origens dos trens. Esta movimentação nem sempre é viável por conta da indisponibilidade de recursos como maquinistas e faixas de circulação. 


\section{CONCLUSÕES}

O presente trabalho apresentou um modelo de alocação de uma frota de locomotivas para um conjunto de trens de carga através de um procedimento otimização com foco na redução de dois dos principais custos da operação de locomotivas. Além disso, buscou-se também a redução da emissão de $\mathrm{CO}_{2}$ de modo a se obter ganhos ambientais com a proposta elaborada. Este é um problema de grande relevância, como mostrado na revisão sobre o tema, visto seu potencial de ganhos, sua complexidade e a falta de ferramentas que garantam que as locomotivas são alocadas da maneira mais eficiente nas composições ferroviárias.

O modelo proposto apresentou ganhos quando comparado com a prática atual para alocação das locomotivas adotada pela empresa estudada. Foram levados em consideração dados reais de alocação de locomotivas para uma semana de transporte de cargas. A economia para uma semana dos custos de combustível e manutenção das locomotivas foi da ordem de $10 \%$, representado mais de 100 mil reais de redução de custos. Além disso, o modelo de alocação permitiu uma redução da quantidade necessária para a realização do transporte em 13 locomotivas, gerando uma sobra de recursos que pode ser aproveitada para prospecção de novas cargas, manutenções preventivas, entre outras finalidades.

Em termos de comparação do resultado obtido com alguns dos estudos apresentados na revisão bibliográfica, Ziarati et al. (1997) e Ahuja et al. (2005) apresentam redução de custos de aproximadamente 7\% em relação ao método utilizado pela empresa estudada enquanto que Kuo et al. (2007), por sua vez, geram uma redução de cerca de $22 \%$ de custos com o modelo proposto no trabalho.

Em termos de impactos ambientais, o modelo também apresentou ganhos, uma vez que foi possível reduzir o consumo de óleo diesel pelos trens em, aproximadamente, 7\%. Essa redução gerou uma diminuição da emissão de mais de 56 toneladas de $\mathrm{CO}_{2}$ na atmosfera na semana analisada, equivalente ao plantio de aproximadamente 343 árvores.

Outro ponto de destaque do modelo apresentado diz respeito à representatividade de cada um dos custos considerados (de combustível e de manutenção) no custo total do transporte. Pôde-se perceber que o custo de combustível é o maior impactante no resultando global, o que ajuda a direcionar os esforços para alternativas de melhoria da eficiência energética quando se busca a redução de custos e, consequentemente, melhoria no impacto ambiental e da competitividade do transporte ferroviário de cargas.

Além disso, a realização de ajustes na disponibilidade inicial das locomotivas permitiu um incremento nos ganhos do modelo, otimizando tanto os custos do transporte, quanto a emissão de $\mathrm{CO}_{2}$ e o número de locomotivas utilizadas. Apesar de nem sempre ser possível a movimentação prévia dos recursos, esse resultado pode ser utilizado como um direcionamento para as equipes de programação dos trens na busca pelo melhor resultado possível para a alocação de locomotivas. Por fim, uma vez tendo sido levantados os dados que suportam o modelo, este é de fácil aplicação no contexto prático e apresenta ganhos diretos na operação de trens, podendo, inclusive, ser expandido para outros tipos de trens, possuindo estes grade horária de circulação ou não. 


\section{REFERÊNCIAS}

AAR. American Association of Railroads. Rail Cost Adjustment Fator: 2018 Q3. Washington: AAR, 2018.

AHUJA, R. K.; LIU, J.; ORLIN, J. B.; SHARMA, D.; SHUGHART, L. A.. Solving real-life locomotive-scheduling problems. Transportation Science, v.39, n.4, p.503-517, 2005. DOI: http://doi.org/10.1287/trsc.1050.0115

ANTT. Agência Nacional De Transportes Terrestres. 10 Inventário Nacional de Emissões Atmosféricas do Transporte Ferroviário de Cargas. Brasília: ANTT, 2012.

ARAGÓN, C. S.; PAMPLONA, E.; MEDINA, J. R. V.. Identificação de investimentos em eficiência energética e sua avaliação de risco. Gestão \& Produção, v.20, n.3, p.4-16, 2013. DOI: http://doi.org/10.1590/S0104530X2013000300003

ARAÚJO, A. C. P.. Como comercializar créditos de carbono. São Paulo: Trevisan, 2006.

ARONSSON, M.; KREUGER, P.; GJERDRUM, J.. An efficient MIP model for locomotive routing and scheduling. WIT Transactions on the Built Environment, v.114, p.963-973, 2010. DOI: http://doi.org/10.2495/CR100871

AZANOV, V. M.; BUYANOV, M. V.; GAYNANOV, D. N.; IVANOV, S. V.. Algorithm and software development to allocate locomotives for transportation of freight trains. Bulletin of the South Ural State University, Series: Mathematical Modelling, Programming and Computer Software, v.9, n.4, p.73-85, 2016. DOI: https://doi.org/10.14529/mmp160407

CASSEMIRO, S. L. S.. Procedimento para Planejamento de Alocação de Locomotivas. Dissertação (Mestrado em Engenharia de Transportes) - Instituto Militar de Engenharia, Rio de Janeiro, 2017.

CASSEMIRO, S. L. S.; COSTA, F. A. C. V.. Distribuição otimizada de locomotivas para ganho de eficiência energética. In: CONGRESSO NACIONAL DE PESQUISA EM TRANSPORTE DA ANPET, 29. Anais. Ouro Preto: 2015.

CNT. Confederação Nacional do Transporte. Anuário CNT do Transporte: Estatísticas Consolidadas. Brasília: CNT, 2017.

CNT. Confederação Nacional do Transporte. 0 sistema ferroviário brasileiro. Brasília: CNT, 2013.

D'AGOSTO, M. A.. Transporte, uso de energia e impactos ambientais: uma abordagem introdutória. Rio de Janeiro: Elsevier, 2015.

EPA. Environmental Protection Agency. Emission Factors for Locomotives. Washington: EPA, 2009.

GHOSEIRI, K.; GHANNADPOUR, S. F.. A hybrid genetic algorithm for multi-depot homogenous locomotive assignment with time windows. Applied Soft Computing Journal, v.10, n.1, p.53-65, 2010. DOI: https://doi.org/10.1016/j.asoc.2009.06.004
IEMA. Instituto de Energia e Meio Ambiente. Emissões de GEE do setor de energia, processos industriais e uso de produtos. São Paulo: IEMA, 2016.

IVANOV, S. V.; KIBZUN, A. I.; OSOKIN, A. V.. Stochastic optimization model of locomotive assignment to freight trains. Automation and Remote Control, v.77, n.11, p.19441956, 2016. DOI: https://doi.org/10.1134/S0005117916110059

JAUMARD, B.; TIAN, H.. Multi-column generation model for the locomotive assignment problem. OpenAccess Series in Informatics, n.6, 2016. DOI:

https://doi.org/10.4230/OASIcs.ATMOS.2016.6

KUO, C. C.; NICHOLLS, G. M.. A mathematical modeling approach to improving locomotive utilization at a freight railroad. Omega, v.35, n.5, 472-485, 2007. DOI: https://doi.org/10.1016/i.omega.2005.09.003

MACHADO, M. N.. Pesquisa Operacional Aplicada ao Processo de Planejamento e Programação da Operação do Transporte Ferroviário de Carga. Monografia (Especialização em Transporte Ferroviário de Cargas) - Instituto militar de Engenharia, Rio de Janeiro, 2006.

PEREIRA, O. C.. Soluções de otimização da eficiência energética de uma ferrovia de carga: caso da estrada de ferro Carajás. Tese (Doutorado) - Pontifícia Universidade Católica do Rio de Janeiro, Rio de Janeiro, 2009.

ROUILLON, S.; DESAULNIERS, G.; SOUMIS, F.. An extended branch-and-bound method for locomotive assignment. Transportation Research Part B: Methodological, v.40, n.5, p.404-423, 2006. DOI: https://doi.org/10.1016/j.trb.2005.05.005

SALUM, L. J. B.. Energia Eficaz. Belo Horizonte: CEMIG, 2005.

SU, R., ZHOU, L., \& TANG, J.. Locomotive schedule optimization for Da-qin heavy haul railway. Mathematical Problems in Engineering, p.1-14, 2015. DOI: https://doi.org/10.1155/2015/607376

TEICHMANN, D.; DORDA, M.; GOLC, K.; BÍNOVÁ, H. Locomotive Assignment Problem with Heterogeneous Vehicle Fleet and Hiring External Locomotives.

Mathematical Problems in Engineering, v.2015, 2015. DOI: https://doi.org/10.1155/2015/583909

THOMÉ, A. M. T.; HOLLMANN, R. L.; CARMO, L. F. R. R. S.. Research synthesis in collaborative planning forecast and replenishment. Industrial Management \& Data Systems, v.114, n.6, p.949-965, 2014. DOI: http://doi.org/10.1108/IMDS-03-2014-0085

VAIDYANATHAN, B.; AHUJA, R. K.; ORLIN, J. B.. The locomotive routing problem. Transportation Science, v.42, n.4, p.492-507, 2008. DOI:

http://doi.org/10.1287/trsc.1080.0244

$\mathrm{XU}, \mathrm{X}$; $\mathrm{LI}, \mathrm{K} .$. Cooperation research for locomotives assignment and train scheduling problems. In: INTERNATIONAL CONFERENCE ON COMPUTER SCIENCE AND 
NETWORK TECHNOLOGY, 4. Anais. Otawa: IEEE Xplore, 2016.

ZHANG, X.; MO, W.; WANG, B.; WANG, F.; GAO, P.. Graph partition based decomposition approach for large-scale railway locomotive assignment. Open Cybernetics and Systemics Journal, v.9, n.1, p.243-252, 2015. DOI: http://doi.org/10.2174/1874110X01509010243
ZIARATI, K., CHIZARI, H., \& MOHAMMADI NEZHAD, A.. Locomotive optimization using artificial intelligence approach. Iranian Journal of Science and Technology, v.29, p.93-105, 2005.

ZIARATI, K.; SOUMIS, F.; DESROSIERS, J.; GÉLINAS, S.; SAINTONGE, A.. Locomotive assignment with heterogeneous consists at CN North America. European Journal of Operational Research, v.97, n.2, p.281-292, 1997. DOI: https://doi.org/10.1016/S0377-2217(96)00198-1

A CBPC - Companhia Brasileira de Produção Científica (CNPJ: 11.221.422/0001-03) detém os direitos materiais desta publicação. Os direitos referem-se à publicação do trabalho em qualquer parte do mundo, incluindo os direitos às renovaç̃ões, expansões e disseminações da contribuição, bem como outros direitos subsidiários. Todos os trabalhos publicados eletronicamente poderão posteriormente ser publicados em coletâneas impressas sob coordenação da Sustenere Publishing, da Companhia Brasileira de Produção Científica e seus parceiros autorizados. Os (as) autores (as) preservam os direitos autorais, mas não têm permissão para a publicação da contribuição em outro meio, impresso ou digital, em português ou em tradução. 